\title{
Fibrolipoma gigante de la mejilla: a propósito de un caso
}

\section{Scivetti $M^{*}$, Di Cosola $M^{*}$, Lo Muzio L***, Pilolli GP*, Maiorano E**, Capodiferro} $\mathrm{S}^{*}$, Vignoletti $\mathrm{F}^{* * * *}$, Favia GF*

\section{RESUMEN}

El fibrolipoma es una neoplasia de origen mesenquimal, rara en la cavidad oral, representando el $1 \%$ de todos los tumores benignos orales. Es una variante histológica del lipoma simple, que normalmente se manifiesta a nivel de la mucosa oral, suelo de la boca, lengua y labios. La etiología suele ser traumática cuando afecta localizaciones extraorales, mientras se considera idiopática al manifestarse a nivel bucal. En este artículo se presenta un raro caso de fibrolipoma de mejilla, y se discuten los aspectos diagnósticos, histo-patológicos y de tratamiento de acuerdo a la literatura.

Palabras clave: Lipoma, neoplasia benigna, fibrolipoma, mejilla.

Aceptado para publicación: Octubre 2005

* Departamento de Ciencias Odontológicas y Cirugía

** Departamento de Anatomía Patológica y Cirugía. Universidad de Bari, Italia

*** Departamento de Ciencias Odontológicas y Cirugía. Universidad de Foggia, Italia

**** Departamento de Medicina y Cirugía Bucofacial. Facultad de Odontología. Universidad Complutense, Madrid, España.

Scivetti M, Di Cosola M, Lo Muzio L, Pilolli GP, Maiorano E, Capodiferro S, Vignoletti F, Favia GF. Fibrolipoma gigante de la mejilla: a propósito de un caso. Av. Odontoestomatol 2006; 22, 1:33-36.

\section{INTRODUCCIÓN}

El lipoma es una neoplasia mesenquimal de los tejidos blandos, rara en la cavidad oral, y que representa el $1 \%$ de todos los tumores orales (1-3).

El fibrolipoma es una variante histológica del lipoma simple: la literatura evidencia una incidencia parecida entre las dos formas, aunque algunos estudios han observado un mayor número de casos de lipomas simples en la cavidad oral. El lipoma oral afecta primariamente la mucosa bucal, el fondo de la boca, lengua y labios (3).

Aunque el trauma se considera un factor etiológico de los lipomas que afectan otras localizaciones ex- traorales, la etiología de los lipomas orales es desconocida. Raramente pueden formar parte de lipomatosis múltiples (2).

Los fibrolipomas intraorales aparecen como nódulos submucosos de forma circular u ovoide, que provocan una tumoración localizada de crecimiento lento; otras posibles manifestaciones clínicas son en forma de masas libres blandas, sésiles o a veces pediculadas. Una fina mucosa normotrófica cubre la neoplasia con un patrón vascular superficial que, debido al alto contenido en tejido adiposo, a veces aparece de un color verde-amarillo. Por la ausencia de síntomas clínicos como dolor o sangrado y debido a su comportamiento benigno, los pacientes no acuden al clínico hasta que la neoplasia alcance cier- 
tas dimensiones y pueda provocar dolor durante la masticación.

\section{CASO CLÍNICO}

Se presenta el caso de un paciente varón de 57 años referido al Departamento de Medicina Oral por presentar una tumoración a nivel de la mejilla derecha. El paciente se le diagnosticó la tumoración el año anterior. Desde entonces el crecimiento fue lento pero constante.

Presenta una historia de ex fumador (20 cigarrillos/ día durante 30 años) y negativa al abuso de alcohol. La historia médica refiere hipertensión arterial durante 10 años, ninguna cirugía de cuello o cabeza previas ni tratamiento radiante. La exploración clínica revela una tumoración dura de 4 centímetros de diámetro en la mucosa retrocomisural de la mejilla derecha (Fig.1).

El aspecto macroscópico se presentaba como una neoformación pediculada y recubierta por una fina capa de epitelio vascularizado. La superficie no era homogénea, presentando áreas de ulceración intercaladas con áreas de hiperqueratosis, probablemente relacionadas con el trauma por masticación. El paciente podía evertir la neoplasia fuera de la cavidad bucal.

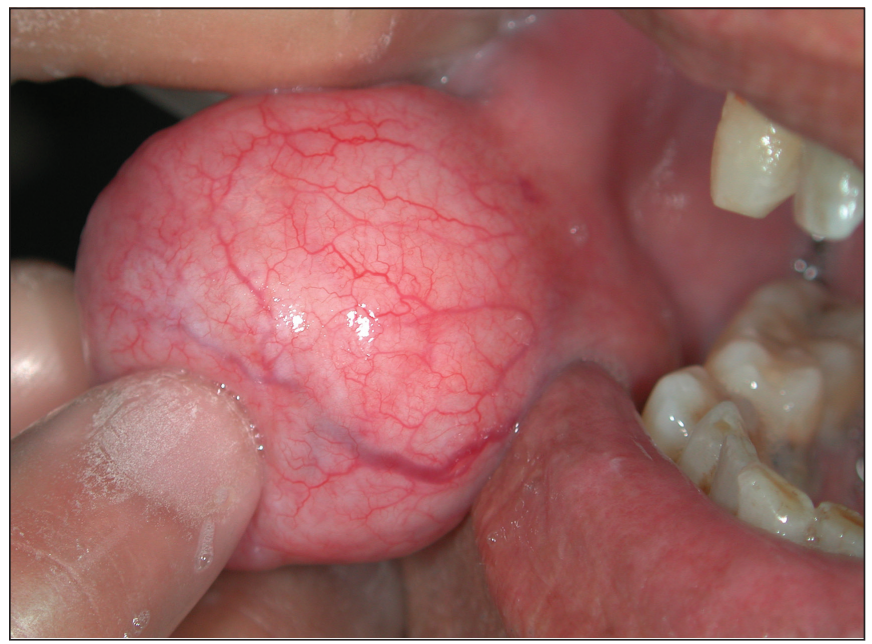

Fig.1. Tumoración dura de 4 centímetros de diámetro en la mucosa retro comisural de la mejilla derecha.

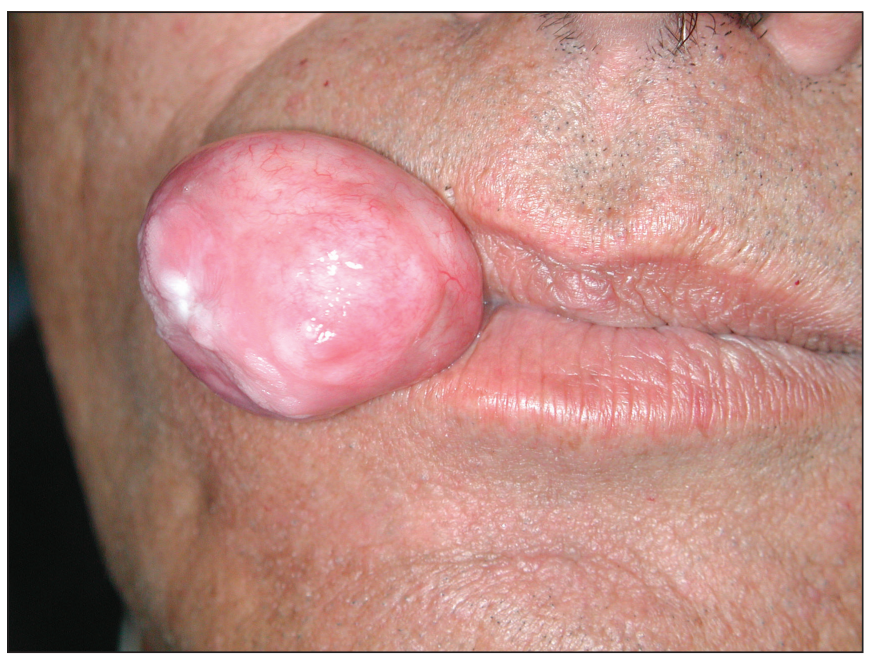

Fig.2. El paciente podía evertir la neoplasia fuera de la cavidad bucal. El epitelio presenta áreas de ulceración intercaladas con áreas de hiperqueratosis, probablemente relacionadas con el trauma por masticación.

El tratamiento quirúrgico consistió en una escisión de la masa tras una incisión elíptica a la base del pedículo que apareció fibroso y escaso en vasos. Se consiguió cierre por primera intención sin dificultades con el fin de garantizar un postoperatorio sin complicaciones para el paciente. Se seccionó el espécimen (Fig. 3), se fijó en solución tampón al 10 \% de formalina y se preparó para el examen microscópico.

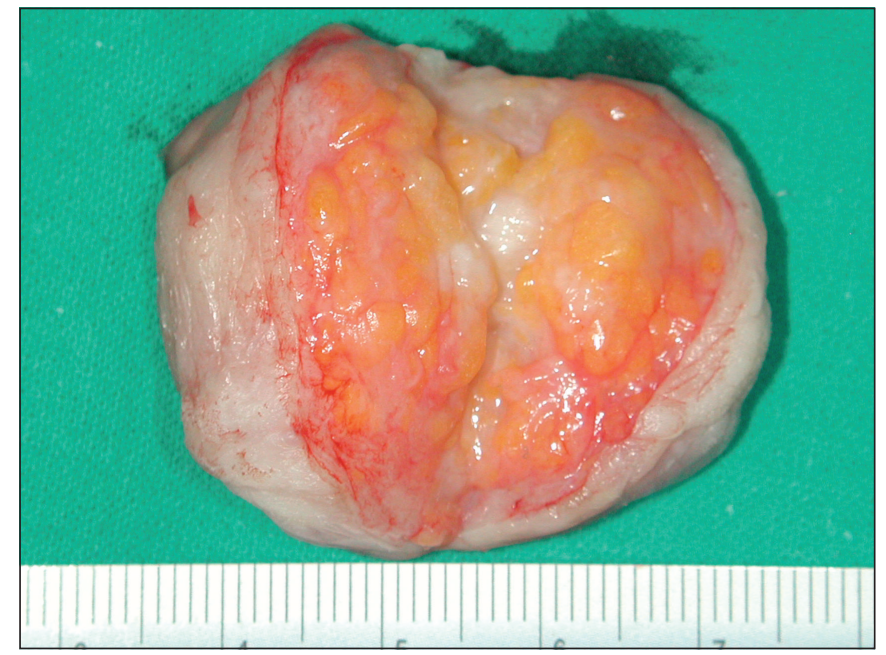

Fig.3. Características del espécimen. 
Los datos histológicos revelaron un epitelio normal, con raras áreas de hiperqueratinización, que recubría tejido adiposo maduro intercalado por anchas bandas y fascículos de conectivo denso. Los limites externos eran delimitados por la presencia de una cápsula fibrosa (Fig. 4). La diagnosis del patólogo fue de fibrolipoma benigno. Tres años tras la cirugía no hubo ningún signo de recidiva.

\section{DISCUSIÓN Y CONCLUSIONES}

Los lipomas son raras neoplasias de la boca de origen mesenquimal, con un $15-20 \%$ de los casos que afectan la región de cuello y cabeza y sólo un $1-4 \%$ que afectan la cavidad oral. Representan el 0,1-5\% de todos los tumores benignos de la boca. Las localizaciones predominantes son mucosa bucal, fondo de la boca, lengua y labios (2). La localización de la mejilla se considera bastante rara, y en particular en la zona posterior a la comisura labial, donde originan desde el tejido adiposo de Bichat. Esta neoplasia suele ser de crecimiento lento y de comportamiento benigno, como demostrado por la tasa muy baja de recurrencia (3). Histológicamente se clasifican en clásicos y variantes mixtas (fibroso, angiomatoso, miocelular y de células "spindle". El lipoma normalmente consiste de células adiposas circundadas por cápsula fibrosa. Los casos orales son más frecuentes en varones mien-

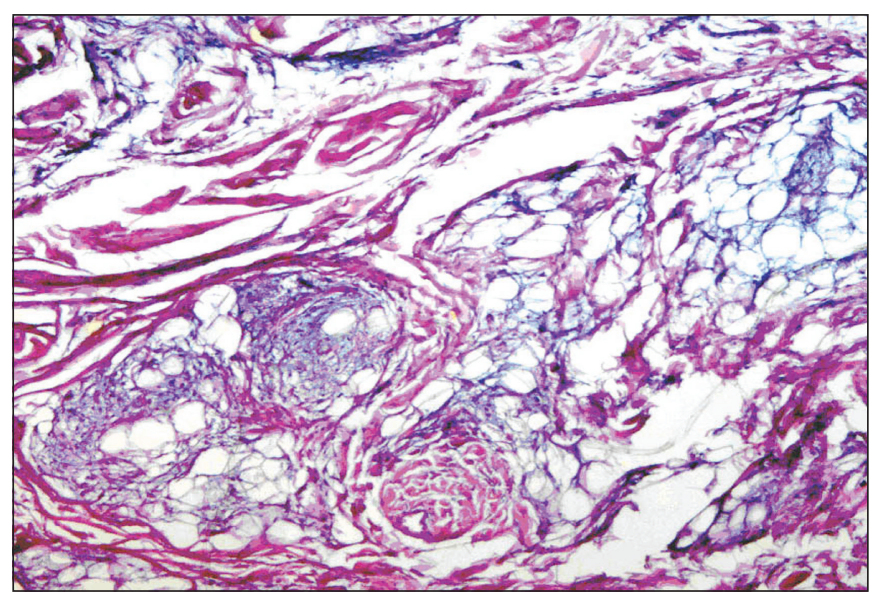

Fig.4. Los datos histológicos revelaron tejido adiposo maduro intercalado por anchas bandas y fascículos de conectivo denso. (Ematosina/ Eosina, 20X). tras que los casos con localización extraoral son 2 veces más comunes en mujeres. Los fibrolipomas difieren del lipoma clásico por el tejido adiposo maduro intercalado con fascículos de tejido conectivo. El lipoma a células "spindle", fue descrito por primera vez por Enzinger y Harvey en 1975. Consiste en un número variable de células spindle, adipocitos y fascículos de colágeno y matriz intersticial mixoide. Las células spindles no se marcan con la proteína S-100, actina o desmina a del músculo liso. Los angiolipomas y miolipomas son infrecuentes en la cavidad oral; el angiolipoma, al contrario de los lipomas convencionales es a veces sintomático (3-6). Se debe considerar la variante "lipomalike" del liposarcoma, por las características clínicas y patológicas parecidas. Con respecto a la actividad de proliferación, la literatura reporta diferencias en PCNA y Ki67 en distintos grupos histológicos benignos; normalmente, los fibrolipomas presentan una tasa de proliferación mas elevada con respecto a las de las de más variantes; consecuentemente PCNA y Ki67 son más elevados y representan un patrón de crecimiento más rápido, que pero no refleja un peor pronóstico o una mayor posibilidad de recidiva tras el tratamiento quirúrgico (3).

La diagnosis diferencial incluye distintos tumores mesenquimales benignos como el fibroma, lipomas simples, tumores de las glándulas salivales minores benignos o malignos. De acuerdo a la literatura, es difícil evaluar la real incidencia de esta neoplasia debido a la ausencia de sintomatología y a su crecimiento lento, que hacen que el paciente acuda al clínico solo en una fase avanzada sintomática o por problemas estéticos o funcionales.

El tratamiento quirúrgico requiere una atención particular en la escisión a la hora de enfrentarse a zonas del distrito orofacial, sobretodo cuando es importante valorar modificaciones estéticas o funcionales causadas por la terapia. La posibilidad de recidiva, aunque rara, está íntimamente relacionada con el estado de los márgenes durante la cirugía y parece obvio que una escisión completa es más fácil cuando el cirujano se enfrenta con una lesión pequeña. En este caso, la presencia de un pedículo hizo más fácil el tratamiento quirúrgico; si consideráramos un abordaje completo de una masa de estas dimensiones 
en forma sésil, podría tener repercusiones estéticas y funcionales importantes.

Como conclusión es importante intervenir en una fase precoz de la neoplasia para limitar la morbilidad postquirúrgica

\section{BIBLIOGRAFÍA}

1. Kiehl RL. Oral fibrolipoma beneath complete mandibular denture. JADA 1980 Apr; 100(4):5612.

2. Ghandour K, Issa M. Lipoma of the floor of the mouth. Oral Surg Oral Med Oral Pathol Jan 1992; 73:59-60.

3. Fregnani ER, Pires FR, Falzoni R, Lopes MA, Vargas PA. Lipomas of oral cavity: clinical findings, histological classification and proliferative activity of 46 cases. Int J Oral Maxillofac Surg 2003; 32: 49-53.
4. Graham GS, Brannon RB, Houston GD. Fibrolipoma of the gingiva. A case report. $J$ Periodontol Feb 1998; 59(2): 118-20.

5. Oliveros-chaparro C, Borgarin-Rdriguez J, Sanchez-Mendez M. Giant fibrolipoma of the floor of the mouth. Presentation of a clinical case. Invest Clin Jun 2001; 42(2): 147-52.

6. Robbins SL, Cotran RS, Kumar V. Pathological basis of disease. 5th ed. Philadelphia: W. B. Saunders Company, 1994; 1416.

\section{CORRESPONDENCIA}

Dr. Michele Di Cosola

Department of Dental Sciences

University of Bari - piazza Giulio Cesare, 11

70124 Bari - Italy

tel.: +39 (080) 5478173

fax: + 39 (080) 5478743

e-mail: dicosola@libero.it 